2. Adelroth E, Rak S, Haahtela $\mathrm{T}$, et al. Recombinant humanized $\mathrm{mAb}-\mathrm{E} 25$, an anti-IgE $\mathrm{mAb}$, in birch polleninduced seasonal allergic rhinitis. J Allergy Clin Immunol 2000; 106: 253-259.
3. Noga O, Hanf G, Kunkel G. Immunological and clinical changes in allergic asthmatics following treatment with omalizumab. Int Arch Allergy Immunol 2003; 131: 4652 .

\title{
Alveolization: does "A" stand for appropriate morphometry?
}

\section{To the Editor:}

In a recent issue of the European Respiratory Journal, HIND AND MADEN [1] reported a series of well-designed experiments conducted to further enlighten the role of retinoic acid in alveolar regeneration. The ability of retinoic acid to rescue emphysematous lungs, which was initially reported by MASSARO and MASSARO [2], has attracted great attention. However, the potential of retinoic acid is still controversially discussed [3]. Unquestionably, the study of HIND and MADEN [1] added important new data to our knowledge of the role of retinoic acid in alveolization, as was emphasised in an accompanying editorial [4]. In their editorial, TORDAY and REHAN [4] ask "Does "A" stand for alveolization?". Can this question be answered on the basis of the data presented?

In the context of lung development, alveolization is recognised as the process of subdivision of lung saccules by outgrowing secondary septa, which results in an increased number of smaller subunits, now termed alveoli [5]. As a consequence, total alveolar surface area is considerably enlarged. Hence, alveolization is defined by changes in at least three morphologic characters: alveolar size (volume), total alveolar surface area and number of alveoli. Unfortunately, HIND and MADEN [1], in pursuing to demonstrate that retinoic acid induces alveolar regeneration in the adult mouse, used but one single parameter to assess alveolization: the mean chord length $(\mathrm{Lm})$, also called mean linear intercept length.

The $\mathrm{Lm}$ is a measure of the average distance between two intercepts of a test-line, arbitrarily superimposed on parenchymal tissue, with the alveolar walls. No distinction is made between test lines running within alveoli and those crossing alveolar ducts. Hence, changes in Lm may reflect changes in alveoli or in alveolar ducts. In turn, if alterations are present in both alveoli and alveolar ducts, but are of opposite sign, this may not result in any differences in Lm at all. Therefore, determining $\mathrm{Lm}$ provides us with an indicator of changes in airspace (alveoli plus alveolar duct) size, but it cannot be used to assess changes in alveoli with sufficient certainty, as has been emphasised by others [6].

The enthusiastic reader might argue that the authors also presented data on the total alveolar surface area $(\mathrm{Sa})$. However, as was stated in the Material and methods section, this parameter was derived by calculation from $\mathrm{Lm}$ and lung volume according to WeIBEL [7]. Hence, this calculation is based on the inverse relationship between the surface-tovolume ratio $(\mathrm{S} / \mathrm{V})$ of an object and $\mathrm{Lm}$ according to the formula $S / \mathrm{V}=4 / \mathrm{Lm}$. Thus, the total alveolar surface area of the lung is calculated as $\mathrm{Sa}=4 \times \mathrm{Vlung} / \mathrm{Lm}$. As the surface-tovolume ratio strongly depends on the shape of the object measured, the alveolar surface area calculated from Lm may be affected by changes in shape; as was convincingly demonstrated (fig. 4c and $d$ of [1]), there were considerable alterations in alveoli from control as compared with dexamethasone-treated animals. Even if we accept the assumption to be true that alveoli are of equivalent shape irrespective of treatment with disulphiram, dexamethasone or/and retinol, one problem still needs to be addressed. Measurements of Lm were done on sections of lungs embedded into paraffin, whereas organ volume was determined prior to embedding. Paraffin embedment introduces considerable tissue shrinkage, which affects Lm measurements [8], and no details are given how measurements were corrected for tissue shrinkage.

E.R. Weibel and his co-workers and colleagues have greatly advanced the field of quantitative morphology during the last decades. Today, a whole range of stereological tools is available that can readily be applied to the quantification of morphologic characters related to volume and size, surface area and numbers [9-11], and new promising tools are still emerging [12]. Quantitative morphology does no longer need to make assumptions about the shape or other features of structures to be analysed. MASSARO and MASSARO [2], for example, have already demonstrated how the tools of designbased stereology can be implemented into the investigation of alveolization (including the effects of retinoic acid) as well as alveolar regeneration, and how these tools enormously increase the impact of such studies. It is a pity that HIND and MADEN [1], as well as others [3], wasted so much of the inherent power of their beautifully designed experiments by relying on a single (biologically ill-defined) morphometric measurement, the mean linear intercept length. A variety of versatile design-based stereological tools are available today. Researchers in respiratory medicine should no longer hesitate to make their choice to get better answers to questions like "does "A" stand for alveolization?".

\section{H. Fehrenbach}

Clinical Research Group "Chronic Airway Diseases", Clinic of Internal Medicine (Respiratory Medicine), PhilippsUniversity of Marburg, Germany.

\section{References}

1. Hind M, Maden M. Retinoic acid induces alveolar regeneration in the adult mouse lung. Eur Respir J 2004; 23: 20-27.

2. Massaro GD, Massaro D. Retinoic acid treatment abrogates elastase-induced pulmonary emphysema in rats. Nat Med 1997; 3: 675-677.

3. Fujita M, Ye Q, Ouchi H, et al. Retinoic acid fails to reverse emphysema in adult mouse models. Thorax 2004; 59: 224 230.

4. Torday JS, Rehan VK. Does "A" stand for alveolization? Eur Respir J 2004; 23: 3-4.

5. Burri PH. Structural aspects of prenatal and postnatal development and growth of the lung. In: McDonald JA, ed. Lung Growth and Development. New York, Marcel Dekker, Inc., 1997; pp. 1-35.

6. Blanco LN, Massaro GD, Massaro D. Alveolar dimensions and number: developmental and hormonal regulation. Am J Physiol 1989; 257: L240-L247. 
7. Weibel ER. Morphometry of the human lung. Berlin, Springer-Verlag, 1963.

8. Thurlbeck WM. The internal surface area of nonemphysematous lungs. Am Rev Respir Dis 1967; 95: 765-773.

9. Gundersen HJG, Bagger P, Bendtsen TF, et al. The new stereological tools: disector, fractionator, nucleator and point sampled intercepts and their use in pathological research and diagnosis. APMIS 1988; 96: 857-881.

10. Cruz-Orive LM, Weibel ER. Recent stereological methods for cell biology: a brief survey. Am J Physiol 1990; 258: L148-L156.

11. Bolender RP, Hyde DM, Dehoff RT. Lung morphometry: a new generation of tools and experiments for organ, tissue, cell, and molecular biology. Am J Physiol 1993; 265: L521L548.

12. Ochs M, Nyengaard JR, Jung A, et al. The number of alveoli in the human lung. Am J Respir Crit Care Med 2004; 169: $120-124$

\section{From the authors:}

H. Fehrenbach is perfectly correct to point out that the assessment of our data is based only on the measurement of mean chord length ( $\mathrm{Lm})$ and lung volume estimations, whereas "a whole range of stereological tools is available for more detailed anaysis". However, the results were so dramatic that we felt that there was no need for these further, rather esoteric analyses, which would not necessarily have provided any further useful information for understanding the biology of the system. The results were also so clear-cut that errors due to tissue shrinkage (all samples were processed at the same time so this error should be equal anyway) or including the occasional alveolar duct in our computer-based measurements of alveolar Lm (800 measurements per field of view, 30 fields of view per Lm score) were also likely to be insignificant.
It is not these stereological debates that are the source of the controversies about whether or not retinoic acid (RA) induces alveolar regeneration, since the initial positive report by MASSARO and MASSARO [1]. We should instead be concerning ourselves with other biological reasons, such as the extent of initial damage, methods of delivery of RA, the phamacokinetics of RA, times after dosing, age of the animals etc., as potential causes for these glaringly opposite reports of "RA induces alveolar regeneration" versus "RA does not induce alveolar regeneration".

Nevertheless, it must be acknowledged, as we do in our paper and as the editorial does, that the most important feature of these regenerated lungs is to determine whether they can or cannot take up oxygen efficiently, that is, whether they are fully functional. This is the missing piece of evidence in these and other experiments determining the forced expiratory volume in one second of the mouse or devising a mini-mouse exercise test. However, it would be very surprising if animals had evolved developmental and regenerative mechanisms that resulted in structurally sound but nonfunctional organs. In the words of the playwrite "A" stands for absolutely (perhaps).

M. Maden*, M. Hind ${ }^{\#}$

*MRC Centre for Developmental Neurobiology, King's College London, and ${ }^{\#}$ Royal Brompton Hospital, London, UK.

\section{References}

1. Massaro GD, Massaro D. Retinoic acid treatment abrogates elastase-induced pulmonary emphysema in rats. Nat Med 1997; 3: 675-677.

\section{Pre- and post-bronchodilator spirometric values and the degree of reversibility in patients with COPD}

\section{To the Editor:}

I thank P. Sterk for an interesting and informative Editorial on the definition and classification of patients with chronic obstructive pulmonary disease (COPD) [1]. However, I cannot help but get the feeling from reading the article that perhaps most, if not all, of the emphasis is placed on post-bronchodilator spirometric values. As pointed out by STERK [1], COPD is a disease where airflow limitation is not fully reversible. Taking this into account, one would intuitively expect that the definition and classification of COPD should at least be based on three criteria: namely, preand post-bronchodilator spirometric values, and the degree of reversibility between the two values; rather than depending solely on post-bronchodilator values. Would it not be meaningless to have just a post-bronchodilator value without its pre-bronchodilator counterpart, as significant reversibility wrongly diagnosed as fixed airflow limitation, as pointed out by STERK [1], may lead to potential over-diagnosis and overestimation of the severity of patients with COPD? It would perhaps be sensible if reporting of future articles relating to research on patients with COPD incorporated both the pre- and post-bronchodilator values, together with the degree of reversibility.

D.K.C. Lee

Dept of Respiratory Medicine, Ipswich Hospital, Ipswich, UK.

\section{References}

1. Sterk PJ. Let's not forget: the GOLD criteria for COPD are based on post-bronchodilator FEV1. Eur Respir J 2004; 23: 497-498.

\section{From the Editor:}

I appreciate D. Lee's comments regarding my editorial on the usage of postbronchodilator spirometry in the Global Initiative for Chronic Obstructive Lung Disease (GOLD) and American Thoracic Society/European Respiratory Society 